\title{
SELECCIÓN DE PROGENITORES, VARIANZAS GENÉTICAS Y HEREDABILIDAD PARA ACUMULACIÓN TEMPRANA DE SACAROSA EN CAÑA DE AZÚCAR
}

\author{
PARENTAL SELECTION, GENETIC VARIANCES AND HERITABILITY FOR EARLY SUGAR CONTENT IN \\ SUGARCANE
}

\author{
Edison Silva Cifuentes ${ }^{1}$, Fernando Castillo González ${ }^{1 \star}$, José D. Molina Galán ${ }^{1}$, Ignacio Benítez Riquelme ${ }^{1}$, Amalio \\ Santacruz Varela ${ }^{1}$ y Raúl Castillo Torres ${ }^{2}$
}

\begin{abstract}
${ }^{1}$ Postgrado en Recursos Genéticos y Productividad, Colegio de Postgraduados. Km 36.5 Carr. México-Texcoco. 56230, Montecillo, Texcoco, Edo. de México. Tel. 01(595) 952-0200, Fax. 01(595) 952-0262. ${ }^{2}$ Centro de Investigación de la Caña de Azúcar del Ecuador. Av. Constitución 100 y Av. Joaquín Orrantia. Guayaquil, Ecuador. Tel 00(593) 85164222.
\end{abstract}

* Autor para correspondencia (fcastill@colpos.mx)

\section{RESUMEN}

La siembra de variedades de alto contenido y acumulación temprana de sacarosa en caña de azúcar (Saccharum spp.) permite incrementar los rendimientos de azúcar e iniciar más temprano el periodo de cosecha, con aumentos marginales de los costos de producción. Para identificar variedades de alto contenido de azúcar y estudiar la variabilidad genética y heredabilidad del contenido temprano de sacarosa, se evaluaron dos grupos de variedades; el primero (G1) formado con 20 variedades y el segundo (G2) con 32 . Además, se evaluaron dos grupos de cruzamientos provenientes de G1, 27 que se establecieron con plántulas provenientes de semilla sexual (CR27) y 12 que se establecieron con esquejes (CR12). En los ensayos G1 y G2 se evaluó la sacarosa aparente, solutos totales, pureza y fibra a los 8,10 y 12 meses durante dos cortes; y en CR27 y CR12 a los 12 meses en un solo corte. Se estimaron los componentes de varianza y heredabilidad en sentido amplio $\left(\mathrm{H}^{2}\right)$ en las variedades, y en sentido estrecho en los cruzamientos $\left(h^{2}\right)$. En los dos grupos de variedades, la varianza genética del contenido de azúcar y variables relacionadas tendieron a disminuir a través de las evaluaciones por edad, y la heredabilidad en sentido amplio presentó valores de medios a altos. Al menos 11 variedades presentaron contenidos altos de azúcar, superiores al testigo comercial. En los cruzamientos sembrados con plántulas (CR27), los estimadores de la varianza entre plantas dentro de familias y del error fueron altos, mientras que los valores de heredabilidad en sentido estrecho fueron bajos. En contraparte, en los cruzamientos sembrados con esquejes las varianzas entre plantas y del error fueron bajas y los valores de heredabilidad altos, similares a los obtenidos en los dos grupos de variedades y a los reportados en otras investigaciones. Evaluar esquejes de plantas jóvenes de semilla sexual en caña de azúcar permitiría mejores estimaciones de variación genética y en menos tiempo, lo cual puede acelerar también los procesos de selección.

Palabras clave: Saccharum spp., contenido temprano de azúcar, parámetros genéticos, sacarosa.

\section{SUMMARY}

Cropping sugarcane (Saccharum spp.) varieties with high sucrose content and early accumulation of it, allow increases of sugar yield as well as an earlier start of the harvest season, with marginal increase in the production costs. With the aim of detecting varieties with higher sugar content and to study the genetic variability and heritability of the early sucrose content, two groups of varieties of sugarcane were evaluated: the first one (G1) integrated by 20 , and the second one (G2) by 32 . In addition, two groups of crosses obtained from G1 were evaluated as well: 27 crosses (CR27) planted with seedlings from sexual seeds, and a subset of 12 (CR12) planted with stem cuttings (setts) obtained from those seedlings. For G1 and G2, sucrose, total solutes, purity and fiber content were recorded at 8,10 and 12 months for two harvest seasons; and for CR27 and CR12 the same information was recorded at 12 months in one harvest season. Variance components and broad sense heritability $\left(\mathrm{H}^{2}\right)$ for variety means were estimated for $\mathrm{G} 1$ and $\mathrm{G} 2$, and narrow sense heritability $\left(h^{2}\right)$ was estimated for $\mathrm{CR} 27$ and $\mathrm{CR} 12$. For variety trials, the genetic variance tended to be reduced through harvest time (age), and broad sense heritability showed medium to high values. At least 11 varieties showed higher sugar content than the commercial check. For crosses from sexual seeds (CR27) the within family variance and error variance values tended to be high, which gave relatively low values of narrow sense heritability; meanwhile, in the cross trial planted with setts, the within family variance and error variance values were low and heritability resulted high. The option of obtaining setts from seedlings in a mating design for sugarcane may be less time consuming procces and produce a better genetic variance estimates, which may impact in the same way for selection purposes.

Index words: Saccharum spp., early sugar content, genetic parameters, sucrose.

\section{INTRODUCCIÓN}

Uno de los objetivos principales de los programas de mejoramiento genético de caña de azúcar (Saccharum spp.) es obtener variedades con rendimientos altos de azúcar mediante el incremento del contenido de sacarosa o del rendimiento de caña. El mejoramiento del aumento del contenido de sacarosa es altamente atractivo porque permite incrementar los ingresos con un aumento marginal en los costos de producción, cosecha, transporte y molienda (Jackson, 2005; Aitken et al., 2006). Estimaciones efectuadas en la industria azucarera australiana muestran que al mejorar el contenido de azúcar se obtiene una rentabilidad 
1.8 veces mayor que la lograda con el aumento del rendimiento de caña, sobre la base de una misma cantidad de azúcar producida. A pesar de esto y de la alta heredabilidad que presenta el contenido de azúcar, ha habido un limitado progreso en el mejoramiento de este carácter en los últimos 40 años (Jackson, 2005). Debido a que el contenido de azúcar en la caña al inicio de la cosecha presenta valores bajos, es conveniente disponer de variedades con alto contenido y acumulación temprana de sacarosa (Cox et al., 1990; Singh y Singh, 2004; Wagih et al., 2004). Variedades de este tipo permiten iniciar la cosecha más temprano y alargar dicho periodo, con una utilización más eficiente de la infraestructura y equipo para la cosecha, transporte y molienda, y así lograr mayor rentabilidad para la industria (Jackson y Morgan, 2003).

Estimaciones precisas de parámetros genéticos tales como componentes de varianza y heredabilidad en sentido estrecho para características económicamente importantes de las poblaciones en los programas de mejoramiento, permiten calcular índices óptimos de selección, maximizar las tasas de ganancia genética y escoger el sistema de selección más adecuado (Milligan et al., 1990). El contenido de azúcar y sus variables relacionadas: pol (sacarosa aparente), grados brix (solutos totales) y pureza (relación entre el contenido de sacarosa y solutos totales en jugo) presentan una alta proporción de la varianza genética total bajo control genético aditivo; además, las heredabilidades en sentido amplio y estrecho son altas (Cox et al., 1990; Singh y Singh, 2004; Wagih et al., 2004).

Lo anterior indica que la selección de progenitores con altos contenidos de azúcar debe ser la base para la generación de progenies con esa característica (Jackson, 2005). Estudios con marcadores moleculares que involucran mapeo de QTLs (quantitative trait loci), sugieren que el contenido de sacarosa es controlado por un alto número de genes, cada uno con efecto pequeño (Aitken et al., 2006). Esto significa que hay una baja probabilidad de obtener progenies con la mejor combinación de alelos en un solo ciclo de cruzamientos, y que la selección recurrente es la alternativa más adecuada para mejorar el contenido de azúcar. $\mathrm{Al}$ respecto, en un estudio del banco de germoplasma del Centro de Investigación de la Caña de Azúcar del Ecuador (CINCAE), se observaron diferencias importantes entre accesiones para contenido de sacarosa.

Sobre esta base, los objetivos del presente trabajo fueron identificar variedades con alto contenido y acumulación temprana de azúcar, y generar cruzamientos para explorar progenitores con alto y bajo contenido de sacarosa, a fin de estudiar la varianza genética y sus componentes, y la heredabilidad en sentido estrecho del contenido y acumulación temprana de sacarosa, con propósitos de selección.

\section{MATERIALES Y MÉTODOS}

$\mathrm{Al}$ tomar como antecedente la evaluación de variedades del banco de germoplasma del CINCAE para contenido de sacarosa y rendimiento de caña, en este estudio se formaron dos grupos de variedades. El primero (G1), integrado por 16 variedades de alto y tres de bajo contenido de sacarosa, más la variedad 'Ragnar' (sembrada en $60 \%$ del área cañera en Ecuador). El segundo grupo (G2) incluyó 26 variedades con alto y dos con bajo contenido de sacarosa, así como dos clones promisorios del programa de mejoramiento del CINCAE más las variedades 'Ragnar' y 'ECU-01' (primera variedad mejorada ecuatoriana). Las variedades del G1 se evaluaron en CINCAE en los ciclos o cortes 2006-2007 y 2007-2008. Las variedades de G2 se evaluaron en los ingenios San Carlos y Valdez de Ecuador, en los cortes 20072008 y 2008-2009. Las características principales de los ambientes de evaluación se muestran en el Cuadro 1.

En 2007, las variedades de G1 se sometieron a tratamiento artificial de inducción a floración en una cámara de fotoperiodo; se obtuvieron flores en 13 variedades y se hicieron 27 cruzamientos (CR27) biparentales (un progenitor femenino con uno masculino) que se evaluaron en CINCAE en el ciclo 2008-2009, y 12 de ellos en otro ensayo (CR12). Además, se estableció otro experimento con los 13 progenitores (PROG) y las variedades 'Ragnar' y 'ECU-01', en el mismo lote de evaluación. En todos los experimentos se usó el diseño de bloques completos al azar con tres repeticiones. La parcela experimental estuvo constituida por cuatro surcos de $5 \mathrm{~m}$ de largo y $1.5 \mathrm{~m}$ de separación. La siembra fue manual con esquejes, con una densidad de 12 yemas por metro lineal. En CR12 se obtuvieron esquejes pequeños de plántulas provenientes de semilla sexual, mientras que en CR27 las plántulas se trasplantaron directamente en el campo. En PROG se usaron plántulas provenientes de yemas. En estos dos experimentos se colocaron 11 plantas por surco separadas a $50 \mathrm{~cm}$. La cosecha se efectuó en los meses de julio y agosto, que corresponde a la primera etapa de la cosecha comercial en Ecuador.

En G1 y G2 se tomaron muestras al azar de ocho tallos en los dos surcos centrales a los 8,10 y 12 meses de edad (cosecha), tanto en caña plantilla (primer corte) como en primera soca (segundo corte). Los tallos se desfibraron y se obtuvieron los valores de sacarosa aparente (POL), solutos totales (BRIX) y fibra (FIB), expresados como porcentaje en caña mediante los procedimientos descritos por Fernandes (2003). Además se calculó la pureza (PUR), como el cociente $\mathrm{pol} / \mathrm{brix}$ en jugo expresado en porcentaje. A la cosecha se registró el peso de los tallos de los dos surcos centrales y se calculó el rendimiento de caña en $t_{\text {ha }}{ }^{-1}(\mathrm{TCH})$. En CR27 y CR12 se tomaron seis plantas al azar y en PROG cuatro, de 
Cuadro 1. Características de los ambientes de evaluación en la costa de Ecuador. 2006-2009

\begin{tabular}{|c|c|c|c|c|c|c|c|c|}
\hline Experimento & Localidad & Latitud Sur & $\begin{array}{l}\text { Longitud } \\
\text { Oeste }\end{array}$ & Altitud (m) & $\begin{array}{c}\text { Precipitación } \\
\text { media anual }(\mathrm{mm})\end{array}$ & $\begin{array}{l}\text { Fecha } \\
\text { siembra }\end{array}$ & $\begin{array}{c}\text { Fecha corte } \\
\text { CP }\end{array}$ & $\begin{array}{c}\text { Fecha corte } \\
\text { PS }\end{array}$ \\
\hline G1 & CINCAE & $02^{\circ} 09^{\prime}$ & $79^{\circ} 26^{\prime}$ & 60 & 1630 & $07 / 06$ & $07 / 07$ & $07 / 08$ \\
\hline G2 & Ingenio San Carlos & $02^{\circ} 13^{\prime}$ & $79^{\circ} 24^{\prime}$ & 44 & 1750 & $07 / 07$ & $07 / 08$ & 07/09 \\
\hline G2 & Ingenio Valdez & $02^{\circ} 09^{\prime}$ & $79^{\circ} 36^{\prime}$ & 13 & 1520 & $07 / 07$ & 07/08 & 07/09 \\
\hline CR27, CR12 y PROG & CINCAE & $02^{\circ} 09^{\prime}$ & $79^{\circ} 26^{\prime}$ & 60 & 1630 & $08 / 08$ & $08 / 09$ & - \\
\hline
\end{tabular}

$\mathrm{CP}=$ caña plantilla; $\mathrm{PS}=$ primera soca; $\mathrm{G} 1=$ Grupo $1 ; \mathrm{G} 2=$ Grupo 2; CR27 = 27 cruzamientos; $\mathrm{CR} 12=12$ cruzamientos; PROG = progenitores; CINCAE = Centro de Investigación de la Caña de Azúcar del Ecuador.

los surcos centrales. De cada una de ellas se usaron cuatro tallos para obtener los valores de POL, BRIX, FIB y PUR. En estos tres ensayos, las variables se evaluaron solamente a los 12 meses de edad.

Se hizo el análisis de varianza combinado por corte para cada variable, en cada fecha de evaluación. En G1 se confundieron los efectos de corte y de año, en una sola fuente de variación, corte-año. El modelo lineal usado para el análisis fue: $Y_{i j k}=\mu+R_{j}+V_{i}+R V_{j i}+C_{k}+R C_{j k}+V C_{i k}+$ $E_{i j k}$; donde $Y_{i j k}=$ observación de la variedad $i$ en la repetición $j$ y el corte-año $k ; \mu=$ media general; $R_{j}=$ efecto de la repetición $j ; V_{i}=$ efecto de la variedad $i, R V_{j i}^{j}=$ efecto de interacción entre la repetición $j$ y la variedad $i$ (Error 1$) ; C_{k}$ $=$ efecto del corte-año $k ; R C_{j k}=$ efecto de interacción entre la repetición $j$ y el corte-año $k$ (Error 2); $V C_{i k}=$ efecto de interacción entre la variedad $i$ y el corte-año $k ; E_{i j k}=$ error experimental (Error 3). Para la estimación de los componentes de varianza, todos los efectos fueron considerados aleatorios y no se consideraron los efectos de corte-año y de la interacción repeticiones x corte-año (Cuadro 2), de acuerdo con lo indicado por Cox et al. (1990).

Para G2 también se hizo la confusión de los efectos de cortes y años y se consideraron los efectos de variedades, corte-año y repeticiones como aleatorios, mientras que el efecto de localidades fue considerado fijo. El modelo lineal usado para el análisis fue: $Y_{i j k l}=\mu+L_{l}+R_{j(l)}+V_{i}+V L_{i l}+$ $R V_{j i(l)}+C_{k}+C L_{k l}+R C_{j k(l)}+V C_{i k}+V C L_{i k l}+E_{i j k}$; donde $Y_{i j k l}$ $=$ observación de la variedad $i$ en la repetición $j$ en el corteaño $k$ y en la localidad $l ; \mu=$ media general; $L_{l}=$ efecto de la localidad $l ; R_{j(l)}=$ efecto de la repetición $j$ dentro de la localidad $l$ (Error 1); $V_{i}=$ efecto de la variedad $i, V L_{i l}=$ efecto de la interacción de la variedad $i$ y la localidad $l ; R V_{\text {iicll }}$ $=$ efecto de interacción entre la repetición $j$ y la variedad $i$ dentro de la localidad $l$ (Error 2$) ; C_{k}=$ efecto del corte-año $k$; $C L_{k l}=$ efecto de la interacción corte-año $k$ con la localidad $l ; R C_{j k(l)}=$ efecto de interacción entre la repetición $j \mathrm{y}$ el

Cuadro 2. Esperanzas de cuadrados medios de los análisis de varianza para los grupos de variedades (G1 y G2), cruzamientos (CR27 y CR12) y progenitores (PROG) de caña de azúcar.

\begin{tabular}{clll}
\hline $\mathrm{FV}$ & $\mathrm{ECM}$ & FV & ECM \\
\hline
\end{tabular}

G1

$\begin{array}{ll}\text { Variedades (Var) } & \sigma_{e}^{2}+c \sigma_{e 1}^{2}+r \sigma_{C V}^{2}+r c \sigma_{V}^{2} \\ \text { Error 1 } & \sigma_{e}^{2}+c \sigma_{e 1}^{2} \\ \text { Error 2 } & \sigma_{e}^{2}+v \sigma_{e 2}^{2} \\ \text { Var. x Corte-año } & \sigma_{e}^{2}+r \sigma_{C V}^{2} \\ \text { Error experimental } & \sigma_{e}^{2}\end{array}$

CR27 y CR12

$\begin{array}{ll}\text { Cruzamientos } & \sigma_{w}^{2}+m \sigma_{e}^{2}+r m \sigma_{f}^{2} \\ \text { Error experimental } & \sigma_{w}^{2}+m \sigma_{e}^{2} \\ \begin{array}{l}\text { Individuos dentro de } \\ \text { cruzamientos }\end{array} & \sigma_{w}^{2}\end{array}$

G2

$\begin{array}{ll}\text { Variedades (Var) } & \sigma_{e}^{2}+c \sigma_{e 2}^{2}+r c l \sigma_{V}^{2} \\ \text { Var x Localidad } & \sigma_{e}^{2}+c \sigma_{e 2}^{2}+r c \sigma_{V L}^{2} \\ \text { Error 2 } & \sigma_{e}^{2}+c \sigma_{e 2}^{2} \\ \text { Var. x Corte-año } & \sigma_{e}^{2}+r l \sigma_{V C}^{2} \\ \text { Var. x Corte-a. x Loc. } & \sigma_{e}^{2}+r \sigma_{V C L}^{2} \\ \text { Error experimental } & \sigma_{e}^{2}\end{array}$

PROG

$\begin{array}{ll}\text { Progenitores } & \sigma_{w}^{2}+m \sigma_{e}^{2}+r m \sigma_{P M}^{2} \\ \text { Error experimental } & \sigma_{w}^{2}+m \sigma_{e}^{2} \\ \begin{array}{l}\text { Individuos dentro de } \\ \text { variedades }\end{array} & \sigma_{w}^{2}\end{array}$

FV = fuente de variación; ECM = esperanza de cuadrados medios; G1 = Grupo 1; G2 = Grupo 2; CR27 = 27 cruzamientos; CR12 = 12 cruzamientos; $\mathrm{PROG}=$ progenitores; $\mathrm{c}=$ número de corte-año; $\mathrm{r}=$ número de repeticiones; $\mathrm{v}=$ número de variedades; $1=$ número de localidades; $\mathrm{m}$ = número de muestras. Componentes de varianza $\left(\sigma^{2}\right)$ para variedades $(\mathrm{v})$; corte-año $\mathrm{x}$ variedades $(\mathrm{cV})$; variedades $\mathrm{x}$ localidades $(V L)$; variedades $\mathrm{x}$ corte-año $\mathrm{x}$ localidades $(V C L)$; de familias $(f)$; del progenitor medio $\left(P_{M}\right)$; error $1\left(e_{1}\right)$; error $2\left(e_{2}\right)$; error experimental (e); y entre plantas dentro de familias $\left({ }_{w}\right)$. 
corte-año $k$ dentro de la localidad $l$ (Error 3); $V C_{i k}=$ efecto de interacción entre la variedad $i$ y el corte-año $k ; V C L_{i k l}=$ efecto de interacción entre la variedad $i$, el corte-año $k$ y la localidad $l ; E_{i j k}=$ error experimental. Los componentes de varianza (Cuadro 2) se estimaron para todos los efectos que involucraban variedades (Jackson y McRae, 1998). En los dos grupos, los errores estándar de los componentes se calcularon de acuerdo con Becker (1986); además, se calculó la heredabilidad en sentido amplio $\left(\mathrm{H}^{2}\right)$, para $\mathrm{G} 1$ se obtuvo con base en los valores medios de dos años y tres repeticiones, mientras que para G2 se usaron los valores medios de dos localidades, dos años y tres repeticiones (Holland et al., 2003). La comparación de medias se hizo con la prueba de Tukey $(\mathrm{P} \leq 0.05)$ y se probó el ajuste de las tres medidas $(8$, 10, 12 meses) de cada variable, al modelo de regresión lineal simple (RLS). Se utilizó el paquete estadístico InfoStat (Di Rienzo et al., 2009).

Para CR27 y CR12, el análisis de varianza de cada variable se hizo por separado de PROG, con un modelo aleatorio. En este ensayo no se incluyeron en el análisis las variedades 'Ragnar' y 'ECU-01'. Los componentes de varianza fueron estimados a partir de los cuadrados medios esperados y se muestran en el Cuadro 2. Se calculó la heredabilidad en sentido estrecho $\left(\mathrm{h}^{2}\right)$, mediante la regresión progenie vs. progenitor medio $\left(b_{\mathrm{pPM}}\right)$. Debido a que los cruzamientos biparentales generan familias de hermanos completos, los estimadores de la varianza aditiva $\left(\sigma_{A}^{2}\right)$ y de dominancia $\left(\sigma_{D}^{2}\right)$ alcularon de acuerdo con Falconer y Mackay (1996). Así: $\sigma_{A}^{2}=b_{\mathrm{pPM}} x \quad \sigma_{F}^{2}$ y $\sigma_{D}^{2}=4\left(\sigma_{f}^{2}-1 / 2 \sigma_{A}^{2}\right)$, donde $\sigma_{F}^{2}$ es la varianza fenotípica y $\sigma_{f}^{2}$ la varianza de cruzamientos o familias de hermanos completos. La varianza fenotípica se obtuvo como dos veces la varianza del progenitor medio $\left(\sigma_{P M}^{2}\right)$ Los errores estándar de los estimadores de las varianzas aditiva y de dominancia se calcularon como una función lineal de las varianzas involucradas, al suponer que éstas son independientes (Olofsson, 2005).

\section{RESULTADOS Y DISCUSIÓN}

\section{Grupo 1}

Las varianzas del error en todas las características fueron más importantes y superiores a la varianza de variedades, con excepción de PUR a los 8 meses (Cuadro 3). La varianza genética presentó una tendencia a disminuir a medida que aumentó la edad al corte, en todas las características con excepción de FIB. Esto indica que se pueden discriminar mejor los genotipos para POL, BRIX y PUR, conforme la evaluación sea más temprana. Similares resultados fueron reportados por Cox et al. $(1990,1998)$ y Jackson y Morgan (2003). La interacción variedades x corte-año no fue significativa en los análisis de varianza, y su componente no fue importante; esto coincide con los reportes de Milligan et al. (1990), Cox et al. (1990) y Gravois y Milligan (1992), quienes indican que la interacción más importante es la genética $\mathrm{x}$ ambientes. Los estimadores de heredabilidad presentaron valores de medios a altos, entre 0.49 y 0.87 para POL, de 0.51 a 0.82 en BRIX, entre 0.57 y 0.81 en PUR, y de 0.30 a 0.58 para FIB; éstos se encuentran en valores similares a los observados en los estudios de Cox et al. (1990), Milligan et al. $(1990,2003)$.

Cuadro 3. Componentes de varianza, errores estándar $( \pm)$ y heredabilidad en sentido amplio $\left(\mathrm{H}^{2}\right)$, para sacarosa aparente $(\mathrm{POL})$, cantidad de solutos totales (BRIX), pureza (PUR) y fibra (FIB) registrados en tres fechas de evaluación para 20 variedades de caña de azúcar (G1).

\begin{tabular}{|c|c|c|c|c|c|c|c|c|c|c|c|c|}
\hline \multirow{2}{*}{ Comp. $^{\dagger}$} & \multicolumn{3}{|c|}{ POL (\%) } & \multicolumn{3}{|c|}{ BRIX (\%) } & \multicolumn{3}{|c|}{ PUR (\%) } & \multicolumn{3}{|c|}{ FIB (\%) } \\
\hline & 8 & 10 & 12 & 8 & 10 & 12 & 8 & 10 & 12 & 8 & 10 & 12 \\
\hline \multirow{2}{*}{$\sigma_{V}^{2}$} & 1.14 & 0.82 & 0.56 & 0.66 & 0.54 & 0.48 & 24.39 & 5.92 & 2.98 & 0.66 & 0.76 & 0.16 \\
\hline & \pm 0.45 & \pm 0.31 & \pm 0.31 & \pm 0.27 & \pm 0.21 & \pm 0.26 & \pm 9.53 & \pm 2.38 & \pm 1.52 & \pm 0.35 & \pm 0.54 & \pm 0.18 \\
\hline \multirow{2}{*}{$\sigma_{e 1}^{2}$} & 0.35 & -0.08 & 0.58 & 0.22 & -0.02 & 0.46 & 6.55 & -0.59 & 0.50 & 0.13 & 0.37 & -0.10 \\
\hline & \pm 0.21 & \pm 0.15 & \pm 0.31 & \pm 0.14 & \pm 0.10 & \pm 0.25 & \pm 3.79 & \pm 1.37 & \pm 1.22 & \pm 0.17 & \pm 0.24 & \pm 0.12 \\
\hline \multirow{2}{*}{$\sigma_{e 2}^{2}$} & 0.07 & -0.06 & 0.05 & 0.03 & -0.02 & 0.03 & 1.72 & -0.65 & 0.53 & -0.03 & 0.12 & -0.02 \\
\hline & \pm 0.09 & \pm 0.01 & \pm 0.10 & \pm 0.05 & \pm 0.02 & \pm 0.06 & \pm 1.95 & \pm 0.01 & \pm 0.73 & \pm 0.02 & \pm 0.14 & \pm 0.03 \\
\hline \multirow{2}{*}{$\sigma_{V C}^{2}$} & 0.02 & -0.12 & 0.00 & 0.02 & -0.04 & 0.00 & 1.64 & -0.53 & 0.23 & 0.45 & 1.25 & 0.46 \\
\hline & \pm 0.13 & \pm 0.12 & \pm 0.17 & \pm 0.09 & \pm 0.09 & \pm 0.13 & \pm 2.65 & \pm 1.22 & \pm 1.09 & \pm 0.26 & \pm 0.53 & \pm 0.27 \\
\hline \multirow{2}{*}{$\sigma_{e}^{2}$} & 1.21 & 1.54 & 1.62 & 0.79 & 0.94 & 1.29 & 20.84 & 13.44 & 9.93 & 1.23 & 1.41 & 1.27 \\
\hline & \pm 0.27 & \pm 0.34 & \pm 0.36 & \pm 0.18 & \pm 0.21 & \pm 0.29 & \pm 4.66 & \pm 3.01 & \pm 2.22 & \pm 0.28 & \pm 0.32 & \pm 0.28 \\
\hline \multirow{2}{*}{$\sigma_{F}^{2}$} & 1.56 & 0.94 & 1.14 & 0.93 & 0.66 & 0.93 & 32.82 & 7.28 & 5.26 & 1.14 & 1.86 & 0.54 \\
\hline & \pm 0.59 & \pm 0.50 & \pm 0.60 & \pm 0.36 & \pm 0.32 & \pm 0.48 & \pm 11.74 & \pm 4.24 & \pm 3.23 & \pm 0.54 & \pm 0.86 & \pm 0.45 \\
\hline \multirow{2}{*}{$H^{2 \dagger \dagger}$} & 0.73 & 0.87 & 0.49 & 0.72 & 0.82 & 0.51 & 0.74 & 0.81 & 0.57 & 0.58 & 0.41 & 0.30 \\
\hline & \pm 0.07 & \pm 0.04 & \pm 0.10 & \pm 0.08 & \pm 0.06 & \pm 0.10 & \pm 0.07 & \pm 0.06 & \pm 0.10 & \pm 0.10 & \pm 0.11 & \pm 0.10 \\
\hline
\end{tabular}

${ }^{\dagger}$ Componentes de varianza $\left(\sigma^{2}\right)$ para variedades $(\mathrm{V})$; error $1\left(e_{1}\right)$; error $2\left(e_{2}\right)$; variedad x corte-año $(\mathrm{VC})$; error $(e)$; y varianza fenotípica $(\mathrm{F}) .{ }^{\dagger \dagger} H^{2}=$ Heredabilidad en sentido amplio, para una localidad, dos cortes y tres repeticiones; $\mathrm{c}=$ número de corte-año; $\mathrm{y} \mathrm{r}=$ número de repeticiones. $H^{2}=$ $\frac{\sigma_{V}^{2}}{\sigma_{V}^{2}+\frac{\sigma_{e 1}^{2}}{c}+\frac{\sigma_{e 2}^{2}}{c}+\frac{\sigma_{V A}^{2}}{c}+\frac{\sigma_{e}^{2}}{r c}}$ 
Los promedios de POL de las 20 variedades de G1 a los 8 y 12 meses (Cuadro 4) confirman que la evaluación temprana permite detectar con facilidad las diferencias entre genotipos. Las variedades 'BJ65152' (11.40 \%) y 'CP57-536’ (9.82\%) presentaron los valores más altos y superaron a las demás ( $\mathrm{P} \leq 0.05)$, a los 8 meses; el testigo 'Ragnar' mostró un promedio de $8.33 \%$. A la cosecha no se observaron diferencias entre genotipos. Al probar el ajuste de la acumulación de sacarosa (POL) al modelo de RLS, 11 variedades presentaron coeficientes de regresión significativos con $\mathrm{R}^{2} \geq 0.67$; las ocho variedades restantes mostraron un mejor ajuste al modelo cuadrático (datos no presentados). Estos resultados son congruentes con lo reportado por Wagih et al. (2004), quienes indicaron que el comportamiento para POL, BRIX y PUR de 24 variedades, se describe bien con regresiones cuadráticas, cuando realizaron evaluaciones mensuales desde los 5 hasta los 14 meses. La producción de caña (TCH) fue estadísticamente igual en todos los genotipos, pero las variedades 'BJ65152', 'CP57-536', 'Amarilla' y 'B74132' presentan contenidos de azúcar superiores a la variedad comercial.

\section{Grupo 2}

A diferencia de lo observado en G1, las varianzas de variedades en todas las características evaluadas a los 8 y 10 meses, fueron más importantes y superiores a las varianzas del error e interacciones, pero a los 12 meses fueron meno- res que esos estimadores (Cuadro 5). Al igual que en G1, las varianzas genéticas presentaron la misma tendencia a disminuir a través de las edades de evaluación en todas las características, con excepción de FIB; esto refuerza lo indicado anteriormente, en el sentido de que se puede realizar una selección más eficiente de genotipos para POL, BRIX y PUR, a los 8 meses de edad. Las interacciones variedades $\mathrm{x}$ localidad, variedades $\mathrm{x}$ corte-año y variedades $\mathrm{x}$ corte-año $x$ localidades no fueron significativas en los análisis de varianza, y sus respectivos componentes de varianza $\sigma_{V L}^{2}, \sigma_{V C}^{2}$ y $\sigma_{V C L}^{2}$ no fueron importantes. Los estimadores de heredabilidad presentaron nuevamente valores de medios a altos; para POL variaron entre 0.65 y 0.78 , en BRIX de 0.64 a 0.77 , para PUR entre 0.51 y 0.82 y en FIB de 0.78 a 0.89 ; estos últimos son ligeramente más altos que los estimadores para G1. Todos los estimadores se encuentran en límites similares a los reportados por Cox et al. (1990), Milligan et al. (1990), Gravois y Milligan (1992) y Milligan et al. (2003).

En la evaluación temprana, las variedades 'BJ65152' y 'C85-102' presentaron promedios de POL de 12.73 y 11.41 $\%$, respectivamente, diferentes a los de las demás $(\mathrm{P} \leq 0.05)$; el testigo 'Ragnar' mostró un promedio de 8.38 \% (Cuadro 6). A la cosecha, los promedios de las variedades 'PR1059' (14.23\%), 'B43-62' (14.03 \%), ‘CC85-63' (13.93\%), 'C105173’ (13.91 \%) y 'SP79-2233’ (13.98\%), fueron diferentes

\begin{tabular}{|c|c|c|c|c|c|c|}
\hline Variedad & POL8 (\%) & POL12 (\%) & $\beta$ & Prob. & $\mathrm{R}^{2}$ & $\mathrm{TCH}$ \\
\hline 'Amarilla'† & 9.40 a-c & $12.05 \mathrm{ab}$ & 0.66 & 0.28 & 0.28 & 138.8 \\
\hline ‘B74224’ & 8.83 a-d & $12.30 \mathrm{ab}$ & 0.88 & 0.05 & 0.67 & 99.6 \\
\hline 'B75469' & 9.08 a-d & $11.90 \mathrm{ab}$ & 0.70 & 0.04 & 0.71 & 129.6 \\
\hline ‘B75500’ & 8.47 a-d & $10.60 \mathrm{ab}$ & 0.54 & 0.36 & 0.21 & 86.4 \\
\hline 'BJ65152’† & $11.40 \mathrm{a}$ & $12.30 \mathrm{ab}$ & 0.23 & 0.42 & 0.17 & 157.2 \\
\hline ‘BJ6811’ & 8.52 a-d & $11.95 \mathrm{ab}$ & 0.86 & 0.02 & 0.79 & 129.8 \\
\hline 'B74132’† & 9.38 a-c & $14.05 \mathrm{a}$ & 1.16 & 0.02 & 0.77 & 142.3 \\
\hline ‘C1051-73’’ & 8.42 a-d & $13.30 \mathrm{ab}$ & 1.23 & 0.01 & 0.84 & 107.4 \\
\hline${ }^{` C C} 85-63^{\prime}$ & 8.58 a-d & $12.80 \mathrm{ab}$ & 1.06 & 0.04 & 0.69 & 129.8 \\
\hline ‘CC85-92’ & 8.22 a-d & $10.45 \mathrm{ab}$ & 0.55 & 0.18 & 0.39 & 104.9 \\
\hline ‘CC $87-474{ }^{\prime \dagger}$ & $7.50 \quad b-d$ & $12.15 \mathrm{ab}$ & 1.16 & 0.02 & 0.76 & 135.2 \\
\hline ‘CC89-2000’ & 9.18 a-d & $10.35 \mathrm{~b}$ & 0.29 & 0.52 & 0.11 & 122.3 \\
\hline${ }^{\prime} \mathrm{Co} 976^{\prime \dagger}$ & $5.92 \mathrm{~d}$ & $12.30 \mathrm{ab}$ & 1.59 & 0.00 & 0.91 & 132.9 \\
\hline 'CР $57-536^{\dagger}$ & $9.82 \mathrm{ab}$ & $11.60 \mathrm{ab}$ & 0.44 & 0.41 & 0.18 & 97.4 \\
\hline 'F140’ & 9.27 a-d & $11.05 \mathrm{ab}$ & 0.45 & 0.33 & 0.24 & 98.4 \\
\hline${ }^{\prime} \mathrm{MZC} 74-275^{\prime \dagger}$ & 7.27 b-d & $13.00 \mathrm{ab}$ & 1.44 & 0.03 & 0.72 & 128.6 \\
\hline ‘Ragnar’ (TC) & 8.33 a-d & $11.70 \mathrm{ab}$ & 0.85 & 0.08 & 0.59 & 99.5 \\
\hline 'B49119' (TBA) & 8.20 & $11.30 \mathrm{ab}$ & 0.78 & 0.05 & 0.67 & 94.0 \\
\hline ‘C132-81’ (TBA) & 6.42 & $11.55 \mathrm{ab}$ & 1.29 & 0.01 & 0.86 & 120.7 \\
\hline 'CR69-177’ (TBA) ${ }^{\dagger}$ & 7.55 & $10.20 \mathrm{~b}$ & 0.39 & 0.69 & 0.04 & 87.4 \\
\hline DSH & 3.03 & 3.65 & & & & 101.6 \\
\hline
\end{tabular}


Cuadro 5. Componentes de varianza, errores estándar $( \pm)$ y heredabilidad en sentido amplio $\left(\mathrm{H}^{2}\right)$ de sacarosa aparente $(\mathrm{POL})$, solutos totales (BRIX), pureza (PUR) y fibra (FIB) en tres fechas de evaluación para 32 variedades de caña de azúcar (G2).

\begin{tabular}{|c|c|c|c|c|c|c|c|c|c|c|c|c|}
\hline \multirow{2}{*}{ Comp. $^{\dagger}$} & \multicolumn{3}{|c|}{ POL (\%) } & \multicolumn{3}{|c|}{ BRIX (\%) } & \multicolumn{3}{|c|}{ PUR (\%) } & \multicolumn{3}{|c|}{ FIB (\%) } \\
\hline & 8 & 10 & 12 & 8 & 10 & 12 & 8 & 10 & 12 & 8 & 10 & 12 \\
\hline \multirow[t]{2}{*}{$\sigma_{V}^{2}$} & 1.12 & 1.06 & 0.59 & 0.71 & 0.67 & 0.49 & 16.16 & 9.18 & 2.74 & 1.26 & 1.12 & 1.38 \\
\hline & \pm 0.29 & \pm 0.28 & \pm 0.17 & \pm 0.18 & \pm 0.17 & \pm 0.14 & \pm 4.29 & \pm 2.41 & \pm 0.94 & \pm 0.33 & \pm 0.31 & \pm 0.36 \\
\hline \multirow[t]{2}{*}{$\sigma_{V L}^{2}$} & 0.16 & 0.08 & 0.12 & 0.14 & 0.09 & 0.18 & 1.85 & -0.02 & -0.83 & 0.09 & 0.07 & 0.02 \\
\hline & \pm 0.07 & \pm 0.05 & \pm 0.09 & \pm 0.05 & \pm 0.04 & \pm 0.09 & \pm 1.08 & \pm 0.29 & \pm 0.32 & \pm 0.05 & 0.08 & \pm 0.04 \\
\hline \multirow[t]{2}{*}{$\sigma_{e 2}^{2}$} & 0.10 & 0.04 & 0.15 & 0.05 & 0.02 & 0.09 & 2.09 & 1.09 & 2.70 & 0.02 & 0.01 & 0.14 \\
\hline & \pm 0.05 & \pm 0.05 & \pm 0.09 & \pm 0.03 & \pm 0.03 & \pm 0.07 & \pm 0.95 & \pm 0.46 & \pm 0.80 & \pm 0.05 & \pm 0.09 & \pm 0.06 \\
\hline \multirow[t]{2}{*}{$\sigma_{V C}^{2}$} & 0.17 & 0.15 & 0.09 & 0.11 & 0.08 & 0.04 & 3.57 & 1.68 & 1.27 & 0.29 & 0.03 & 0.15 \\
\hline & \pm 0.07 & \pm 0.06 & \pm 0.07 & \pm 0.04 & \pm 0.04 & \pm 0.05 & \pm 1.33 & \pm 0.62 & \pm 0.61 & \pm 0.10 & \pm 0.06 & \pm 0.06 \\
\hline \multirow[t]{2}{*}{$\sigma_{V C L}^{2}$} & 0.22 & 0.18 & 0.16 & 0.11 & 0.12 & 0.18 & 6.48 & 1.07 & 1.99 & 0.40 & -0.13 & 0.22 \\
\hline & \pm 0.10 & \pm 0.10 & \pm 0.13 & \pm 0.06 & \pm 0.07 & \pm 0.12 & \pm 2.50 & \pm 0.68 & \pm 1.09 & \pm 0.15 & \pm 0.08 & \pm 0.10 \\
\hline \multirow[t]{2}{*}{$\sigma_{e}^{2}$} & 0.56 & 0.69 & 1.15 & 0.36 & 0.45 & 0.96 & 10.97 & 5.12 & 7.35 & 0.68 & 1.38 & 0.61 \\
\hline & \pm 0.07 & \pm 0.09 & \pm 0.14 & \pm 0.05 & \pm 0.06 & \pm 0.12 & \pm 1.38 & \pm 0.65 & \pm 0.93 & \pm 0.09 & \pm 0.17 & \pm 0.08 \\
\hline \multirow[t]{2}{*}{$\sigma_{F}^{2}$} & 2.34 & 2.20 & 2.26 & 1.48 & 1.43 & 1.93 & 41.12 & 18.12 & 15.23 & 2.73 & 2.48 & 2.50 \\
\hline & \pm 0.33 & \pm 0.32 & \pm 0.30 & \pm 0.21 & \pm 0.21 & \pm 0.26 & \pm 5.51 & \pm 2.72 & \pm 2.01 & \pm 0.39 & \pm 0.38 & \pm 0.39 \\
\hline \multirow{2}{*}{$H^{2 \dagger \dagger}$} & 0.78 & 0.82 & 0.65 & 0.77 & 0.81 & 0.64 & 0.72 & 0.82 & 0.51 & 0.78 & 0.89 & 0.84 \\
\hline & \pm 0.04 & \pm 0.04 & \pm 0.06 & \pm 0.05 & \pm 0.04 & \pm 0.06 & \pm 0.05 & \pm 0.04 & \pm 0.06 & \pm 0.04 & \pm 0.03 & \pm 0.03 \\
\hline
\end{tabular}

${ }^{\dagger}$ Componentes de varianza $\left(\sigma^{2)}\right.$ para variedades $(v)$; variedades $\mathrm{x}$ localidades $(v)$; error $2\left(e_{2}\right)$; variedad $\mathrm{x}$ corte-año $(v c)$; variedad $\mathrm{x}$ corte-año $\mathrm{x}$ localidad $(v C L)$; y error $(e) .{ }^{\dagger \dagger}$ Heredabilidad en sentido amplio para dos localidades, dos cortes y tres repeticiones; $1=$ número de localidades; $\mathrm{c}=$ número de cortesaño; $\mathrm{r}=$ número de repeticiones ${ }^{\dagger \dagger} H^{2}=\frac{\sigma_{V}^{2}}{\sigma_{V}^{2}+\frac{\sigma_{V L}^{2}}{l}+\frac{\sigma_{e 2}^{2}}{l}+\frac{\sigma_{V C}^{2}}{c}+\frac{\sigma_{V C l}^{2}}{c l}+\frac{\sigma_{e}^{2}}{r c l}}$

a los de las demás variedades $(\mathrm{P} \leq 0.05)$; la variedad 'Ragnar' alcanzó un promedio de $12.22 \%$. En cuanto al ajuste del modelo de RLS para la acumulación de sacarosa (POL), siete variedades presentaron valores de $\mathrm{R}^{2} \geq 0.50$; sin embargo, el coeficiente de regresión fue significativo $(\mathrm{P} \leq 0.05)$ en 29 de los 32 casos. Las variedades restantes mostraron un buen ajuste al modelo cuadrático (datos no presentados). Estos resultados son congruentes con los que encontraron Wagih et al. (2004). En producción de caña (TCH), 27 variedades fueron iguales estadísticamente a la variedad testigo 'Ragnar'; en este grupo sobresalieron las variedades 'BJ65152', 'C85-102', 'PR10-28' 'PR1059', 'B43-62', 'CC85-63', 'C1051-73' y 'SP79-2233', y la variedad 'BJ65152' presentó los valores más altos $(\mathrm{P} \leq 0.05)$ de $\mathrm{POL}$ a los 8 meses en los dos grupos.

\section{Cruzamientos}

La varianza entre plantas dentro de familias $\left(\sigma_{w}^{2}\right)$ fue la más importante para los cruzamientos CR27 y CR12 (Cuadro 7). Esto se debió a que este componente contiene parte de la varianza genética, $1 / 2 \sigma_{A}^{2}+3 / 4 \sigma_{D}^{2}$, más varios componentes de varianza epistática que podrían magnificarlo, y varianza ambiental que corresponde a la generada por la competencia entre individuos de la familia (Hogarth, 1977). En los progenitores este componente no fue importante debido a que sólo contiene la variación ambiental entre plantas genéticamente iguales, por lo que los estimadores son menores. Los componentes $\left(\sigma_{w}^{2}\right)$ de los 12 cruzamientos sembrados con esquejes (CR12) fueron menores que los de CR27; esto podría deberse a que las plantas que se desarrollan de esquejes son más homogéneas que las que provienen de plántulas, en las que la varianza ambiental se incrementa por la competencia entre plantas (Hogarth, 1977). El componente de varianza del error $\left(\sigma_{e}^{2}\right)$ de los 12 cruzamientos fue menor en todos los casos que en los 27 cruzamientos evaluados en plántulas, incluso que los progenitores. En cuanto a la varianza de familias $\left(\sigma_{f}^{2}\right)$, la mayor correspondió a PROG debido a que en ella está contenida toda la varianza genética, mientras que en los cruzamientos sólo es una fracción, $1 / 2 \sigma_{A}^{2}+1 / 4 \sigma_{D}^{2}$ (Hogarth, 1977).

Las estimaciones de la varianza de dominancia resultaron con valores más altos que los de la varianza aditiva en todas las características para CR27 (Cuadro 8). Los valores de heredabilidad en sentido estrecho fueron bajos para POL (0.231) y BRIX (0.291) mientras que para PUR y FIB fueron negativos (equivalentes a cero). Estos resultados deben considerarse con reserva porque las estimaciones de los componentes $\sigma_{w}^{2}$ y $\sigma_{e}^{2}$ resultaron altos debido al tipo de material utilizado en la evaluación (plántulas). En contraparte, en los 12 cruzamientos evaluados en esquejes (CR12), las estimaciones de la varianza aditiva fueron más importantes que los de la varianza de dominancia, y los valores de heredabilidad en sentido estrecho se acercaron a los valores en sentido amplio indicados en G1 y G2, y a los reportados en otros estudios ya mencionados. Esto indica que la evaluación de los cruzamientos con material proveniente de esquejes es un sistema adecuado para evaluar eficientemente cruzamientos de caña, tanto por su mayor precisión como 
Cuadro 6. Promedios de sacarosa aparente (POL) a los 8 y 12 meses de edad, pendiente $(\beta)$ y ajuste al modelo de regresión lineal simple $\left(R^{2}\right)$ y promedios de rendimiento (TCH), para 32 variedades de caña de azúcar (G2).

\begin{tabular}{|c|c|c|c|c|c|c|c|c|c|}
\hline Variedad & POL8 ( & & POL12 ( & & $\beta$ & Prob. & $\mathrm{R}^{2}$ & $\mathrm{TCH}$ & \\
\hline 'Amarilla' & 10.01 & $c-g$ & 13.63 & $a-d$ & 0.90 & $<0.0001$ & 0.47 & 109.3 & $a-c$ \\
\hline ‘B4362’ & 10.48 & $b-f$ & 14.03 & $\mathrm{ab}$ & 0.89 & $<0.0001$ & 0.45 & 98.5 & $a-c$ \\
\hline ‘B49119’ (TBA) & 9.50 & $d-j$ & 11.27 & $\mathrm{f}$ & 0.44 & 0.0095 & 0.18 & 104.4 & $a-c$ \\
\hline 'B74132' & 10.73 & b-e & 13.81 & $a-c$ & 0.77 & 0.0004 & 0.32 & 80.9 & $\mathrm{bc}$ \\
\hline 'B74418' & 9.03 & g-k & 12.33 & $a-f$ & 0.83 & 0.0001 & 0.38 & 118.2 & $\mathrm{a}$ \\
\hline 'B75276' & 10.70 & $b-e$ & 11.84 & $d-f$ & 0.29 & 0.0785 & 0.09 & 75.8 & bc \\
\hline ‘B75469’ & 7.59 & 1 & 12.01 & $c-f$ & 1.10 & $<0.0001$ & 0.47 & 101.5 & $a-c$ \\
\hline 'BJ65152’ & 12.73 & $\mathrm{a}$ & 13.21 & a-e & 0.12 & 0.5499 & 0.01 & 85.6 & $a-c$ \\
\hline ‘BJ6905’ & 8.81 & g-1 & 13.11 & $a-f$ & 1.08 & $<0.0001$ & 0.68 & 92.9 & $a-c$ \\
\hline ‘BRD-8126’ & 9.74 & $c-i$ & 13.18 & $a-f$ & 0.86 & $<0.0001$ & 0.51 & 98.3 & $a-c$ \\
\hline ‘C1051-73’ & 10.69 & b-e & 13.91 & $a-c$ & 0.80 & 0.0001 & 0.37 & 98.9 & $a-c$ \\
\hline ‘C132-81’ (TBA) & 8.16 & j-1 & 11.40 & ef & 0.81 & 0.0001 & 0.38 & 109.7 & $a b$ \\
\hline ‘C1324-74’ & 8.98 & g-1 & 12.06 & $c-f$ & 0.77 & 0.0003 & 0.33 & 86.4 & $a-c$ \\
\hline ‘C72-74’ & 9.85 & $c-h$ & 13.50 & $a-d$ & 0.91 & $<0.0001$ & 0.52 & 86.0 & $a-c$ \\
\hline 'C85-101' & 10.88 & b-d & 12.98 & $a-f$ & 0.53 & 0.0033 & 0.23 & 89.5 & $a-c$ \\
\hline ‘C85-102’ & 11.41 & $\mathrm{ab}$ & 13.32 & $a-d$ & 0.48 & 0.0128 & 0.17 & 89.4 & $a-c$ \\
\hline ‘C86-12’ & 10.68 & b-e & 13.46 & $a-d$ & 0.70 & 0.0005 & 0.30 & 81.3 & $\mathrm{bc}$ \\
\hline ‘CC85-63’ & 10.16 & b-g & 13.92 & $a-c$ & 0.94 & $<0.0001$ & 0.41 & 100.5 & $a-c$ \\
\hline ‘CC85-92’ & 10.11 & b-g & 12.63 & $a-f$ & 0.63 & 0.0018 & 0.25 & 108.7 & $a-c$ \\
\hline ‘CC89-2000’ & 9.97 & c-g & 12.53 & $a-f$ & 0.64 & 0.0021 & 0.25 & 100.3 & $a-c$ \\
\hline ‘CP57-536’ & 10.77 & b-e & 12.14 & $b-f$ & 0.34 & 0.0842 & 0.09 & 85.9 & $a-c$ \\
\hline ‘HJ-5747’ & 9.93 & $c-g$ & 11.35 & ef & 0.35 & 0.0133 & 0.17 & 94.9 & $a-c$ \\
\hline 'PR-1028’ & 11.01 & $\mathrm{bc}$ & 13.79 & $a-c$ & 0.70 & $<0.0001$ & 0.43 & 72.7 & c \\
\hline 'PR-1059’ & 10.51 & $b-f$ & 14.23 & $\mathrm{a}$ & 0.93 & $<0.0001$ & 0.54 & 103.2 & $a-c$ \\
\hline ‘Ragnar’ (TC) & 8.38 & i-1 & 12.22 & $b-f$ & 0.96 & $<0.0001$ & 0.43 & 84.1 & $a-c$ \\
\hline ‘SIE-86’ & 8.48 & h-l & 13.24 & a-e & 1.19 & $<0.0001$ & 0.51 & 90.4 & $a-c$ \\
\hline 'SP79-2233’ & 9.46 & $e-k$ & 13.98 & $a b$ & 1.13 & $<0.0001$ & 0.61 & 94.1 & $a-c$ \\
\hline 'V71-51' & 8.09 & $\mathrm{kl}$ & 12.78 & $a-f$ & 1.17 & $<0.0001$ & 0.61 & 96.7 & $a-c$ \\
\hline 'LHo83-153’ & 10.44 & $b-f$ & 13.25 & $a-e$ & 0.70 & 0.0027 & 0.24 & 76.9 & $\mathrm{Bc}$ \\
\hline ‘ECU-01’ (TC) & 10.20 & b-g & 13.21 & a-e & 0.75 & 0.0014 & 0.26 & 98.4 & $a-c$ \\
\hline 'ECSP2000-179’ & 9.23 & $f-k$ & 12.49 & $a-f$ & 0.82 & 0.0001 & 0.37 & 96.5 & $a-c$ \\
\hline 'ECSP2000-214’ & 9.52 & $d-j$ & 13.64 & $a-d$ & 1.03 & $<0.0001$ & 0.40 & 98.1 & $a-c$ \\
\hline DSH & 1.39 & & 1.91 & & & & & 36.7 & \\
\hline
\end{tabular}

POL8 = POL a los 8 meses; POL12 = POL a los 12 meses; TCH = toneladas de caña ha ${ }^{-1}$; TC $=$ testigo comercial; TBA = testigo de bajo contenido de azúcar. Medias con letras iguales en una columna no son estadísticamente diferentes (Tukey, 0.05). DSH = diferencia significativa honesta.

Cuadro 7. Componentes de varianza, errores estándar ( \pm ) de sacarosa aparente (POL), solutos totales (BRIX), pureza (PUR) y fibra (FIB) para cruzamientos y progenitores de caña de azúcar con acumulación temprana de sacarosa. CINCAE.

\begin{tabular}{|c|c|c|c|c|c|c|c|c|c|c|c|c|}
\hline \multirow{2}{*}{ Comp. $^{\dagger}$} & \multicolumn{3}{|c|}{ POL } & \multicolumn{3}{|c|}{ BRIX } & \multicolumn{3}{|c|}{ PUR } & \multicolumn{3}{|c|}{ FIB } \\
\hline & CR27 & CR12 & PM & CR27 & CR12 & PM & CR27 & CR12 & PM & CR27 & CR12 & PM \\
\hline \multirow[t]{2}{*}{$\sigma_{f}^{2 \dagger \dagger}$} & 0.83 & 0.26 & 1.34 & 0.81 & 0.22 & 1.02 & 2.71 & -0.02 & 4.05 & 1.08 & 1.82 & 1.81 \\
\hline & \pm 0.30 & \pm 0.15 & \pm 0.56 & \pm 0.27 & \pm 0.12 & \pm 0.44 & \pm 1.26 & \pm 0.20 & \pm 1.69 & \pm 0.36 & \pm 0.75 & \pm 075 \\
\hline \multirow[t]{2}{*}{$\sigma_{e}^{2}$} & 0.38 & 0.11 & 0.28 & 0.22 & 0.07 & 0.30 & 3.31 & 0.44 & 0.47 & 0.34 & -0.22 & 0.29 \\
\hline & \pm 0.16 & \pm 0.10 & \pm 0.12 & \pm 0.11 & \pm 0.08 & \pm 0.12 & \pm 1.16 & \pm 0.46 & \pm 0.34 & \pm 0.16 & \pm 0.08 & \pm 0.13 \\
\hline \multirow[t]{2}{*}{$\sigma_{w}^{2}$} & 2.69 & 1.51 & 0.67 & 2.08 & 1.19 & 0.56 & 16.31 & 6.84 & 3.00 & 2.98 & 3.06 & 0.75 \\
\hline & \pm 0.19 & \pm 0.16 & \pm 0.09 & \pm 0.15 & \pm 0.13 & \pm 0.07 & \pm 1.14 & \pm 0.72 & \pm 0.39 & \pm 0.21 & \pm 0.32 & \pm 0.10 \\
\hline
\end{tabular}

CR27 $=27$ cruzamientos; CR12 $=12$ cruzamientos; $\mathrm{PM}=$ progenitor medio. ${ }^{\dagger}$ Componentes de varianza $\left(\sigma^{2)}\right.$ para cruzamientos o familias $(f)$; error $(e)$; entre plantas dentro de familias $\left({ }_{w}\right) .{ }^{\dagger \dagger}$ En PM corresponde a la varianza entre variedades (genética).

por el ahorro en tiempo, pues en un solo año fue posible obtener pequeños esquejes de plántulas de semilla sexual y sembrar el ensayo de evaluación (CR12). En todos los trabajos aquí citados sobre heredabilidad (Hogarth, 1977; Cox et al., 1990; Singh y Singh, 2004; Wagih et al., 2004), se evaluaron plantas reproducidas por esquejes y generalmen- te en el segundo año. Con este sistema de reproducción y mediante una selección temprana para POL y BRIX, entre los 8 y los 10 meses, se puede reducir la duración del primer estado de selección en un año, en vista que éste se siembra con plántulas de semilla sexual y generalmente tiene una duración de dos años, como ocurre en el CINCAE. 
Cuadro 8. Componentes de varianza, heredabilidad en sentido estrecho $\left(h^{2}\right)$ y errores estándar $( \pm)$, de sacarosa aparente (POL), solutos totales (BRIX), pureza (PUR) y fibra (FIB) de cruzamientos y progenitores de caña de azúcar con acumulación temprana de sacarosa. CINCAE.

\begin{tabular}{|c|c|c|c|c|c|c|c|c|}
\hline \multirow{2}{*}{ Comp. $^{\dagger}$} & \multicolumn{2}{|c|}{ POL } & \multicolumn{2}{|c|}{ BRIX } & \multicolumn{2}{|c|}{ PUR } & \multicolumn{2}{|c|}{ FIB } \\
\hline & CR27 & CR12 & CR27 & CR12 & CR27 & CR12 & CR27 & CR12 \\
\hline \multirow{2}{*}{$\sigma_{A}^{2}$} & 0.621 & 1.340 & 0.600 & 1.780 & -0.016 & 1.310 & -0.206 & 7.554 \\
\hline & \pm 1.607 & \pm 1.441 & \pm 1.305 & \pm 1.369 & \pm 3.522 & \pm 0.825 & \pm 2.228 & \pm 4.061 \\
\hline \multirow{2}{*}{$\sigma_{D}^{2}$} & 2.096 & 0.658 & 2.048 & -0.222 & 10.885 & 0.717 & 4.717 & -11.770 \\
\hline & \pm 3.431 & \pm 2.944 & \pm 2.825 & \pm 2.782 & \pm 8.661 & \pm 1.834 & \pm 4.683 & \pm 8.658 \\
\hline \multirow{2}{*}{$\sigma_{F}^{2}$} & 2.687 & 1.762 & 2.060 & 1.645 & 6.893 & 1.873 & 3.593 & 4.463 \\
\hline & \pm 0.583 & \pm 0.454 & \pm 0.464 & \pm 0.423 & \pm 1.499 & \pm 0.708 & \pm 0.763 & \pm 1.042 \\
\hline \multirow{2}{*}{$\mathrm{h}^{2}$} & 0.231 & 0.760 & 0.291 & 0.721 & -0.002 & 0.466 & -0.057 & 1.128 \\
\hline & \pm 0.596 & \pm 0.794 & \pm 0.630 & \pm 0.811 & \pm 0.511 & \pm 0.404 & \pm 0.620 & \pm 0.871 \\
\hline
\end{tabular}

CR27 $=27$ cruzamientos; CR12 $=12$ cruzamientos. ${ }^{\dagger}$ Componentes de varianza $\left(\sigma^{2}\right)$ aditiva $(\mathrm{A})$; de dominancia (D); fenotípica $(\mathrm{F})$.

\section{CONCLUSIONES}

Las variedades 'BJ65152', 'CP57-536', 'Amarilla, 'B74132', 'C85-102,' 'PR10-28, 'PR1059', 'B43-62', 'CC85-63', 'C105173’ y 'SP79-2233' presentan contenidos de sacarosa superiores a los de la varieda comercial 'Ragnar', con rendimientos similares de caña. Por ello podrían usarse como progenitores en un programa de selección recurrente para alto contenido y acumulación temprana de azúcar. La evaluación del POL y BRIX en plantas de caña de 8 meses de edad permite hacer una selección más efectiva de los materiales con altos contenidos y acumulación temprana de sacarosa. La heredabilidad en sentido amplio para POL, BRIX y FIB fue alta en los dos grupos de variedades (G1 y G2) del banco de germoplasma. Para la determinación de componentes de varianza y estimadores de heredabilidad de cruzamientos de caña es más apropiado utilizar esquejes en la siembra de ensayos, porque la producción acelerada de esquejes de plántulas jóvenes de semilla sexual permite hecr estimaciones precisas de componentes de varianza y heredabilidad, y este sistema puede ser útil para reducir la duración del estado inicial de selección.

\section{AGRADECIMIENTOS}

A la Fundación para la Investigación Azucarera del Ecuador (FIADE) por el financiamiento otorgado al presente estudio.

\section{BIBLIOGRAFÍA}

Aitken K S, P A Jackson, L C McIntyre (2006) Genetic quantitative trait loci identified for sugar related traits in a sugarcane (Saccharum spp.) cultivar x Saccharum officinarum population. Theor. Appl. Genet. 112:1306-1317.

Becker W A (1986) Manual of Quantitative Genetics. 4th ed. Academic Enterprises. Pullman, Washington, USA. $174 \mathrm{p}$.
Cox M C, D M Hogarth, R T Mullins (1990) Clonal evaluation of early sugar content. Proc. Aust. Soc. Sugar Cane Tech. 12:90-98.

Cox M C, D R Ridge, B Hussey (1998) Optimum time of harvest for high early-CCS sugar varieties. Proc. Aust. Soc. Sugar Cane Tech. 20:218-223.

Di Rienzo J A, F Casanoves, M G Balzarini, L González, M Tablada, C W Robledo (2009) InfoStat versión 2009. Grupo InfoStat, FCA, Universidad Nacional de Córdoba, Argentina. 336 p.

Falconer D S, T F S Mackay (1996) Introduction to Quantitative Genetics. 4 th ed. Ed. Longman. England. $464 \mathrm{p}$.

Fernandes A C (2003) Cálculos na agroindústria da cana-de-açúcar. 2 ed. Sociedade dos Técnicos Açucareiros e Alcooleiros do Brasil. Piracicaba, São Paulo, Brasil. 240 p.

Gravois K A, S B Milligan (1992) Genetic relationships between fiber and sugarcane yield components. Crop Sci. 32:62-67.

Hogarth D M (1977) Quantitative inheritance studies in sugarcane. III. The effect of competition and violation of genetic assumptions on estimation of genetic variance components. Aust. J. Agric. Res. 28:257-268

Holland J B, W E Nyquist, C T Cervantes-Martínez (2003) Estimating and interpreting heritability for plant breeding: an update. In: Plant Breeding Reviews, Vol. 22. J Janick (ed) John Wiley \& Sons. Hobo-ken, New Jersey, USA. pp:9-112.

Jackson P A (2005) Breeding for improved sugar content in sugarcane. Field Crops Res. 92: 277-290.

Jackson P A, T A McRae (1998) Gains from selection of broadly adapted and specifically adapted sugarcane families. Field Crops Res. 59:151162.

Jackson P A, T E Morgan (2003) Early stage selection for commercial cane sugar (CCS) in sugarcane clones: effects of time of sampling and irrigation. Aust. J. Agric. Res. 54:389-396.

Milligan S B, M Balzarini, W H White (2003) Broad-sense heritabilities, genetic correlations, and selection indices for sugarcane borer resistance and their relation to yield loss. Crop Sci. 43:1729-1735.

Milligan S B, K A Gravois, K P Bischoff, F A Martin (1990) Crop effects on broad-sense heritabilities and genetic variances of sugarcane yield components. Crop Sci. 30:344-349.

Olofsson P (2005) Probability Statistics and Stochastic Processes. John Wiley and Sons. Hoboken, New Jersey. 486 p.

Singh R K, S B Singh (2004) Breeding strategies for commercially elite early maturing varieties of sugarcane (Saccharum Species Complex). Sugar Tech 6:89-92.

Wagih M E, A Ala, Y Musa (2004) Evaluation of sugarcane varieties for maturity earliness and selection for efficient sugar accumulation. Sugar Tech 6:297-304. 